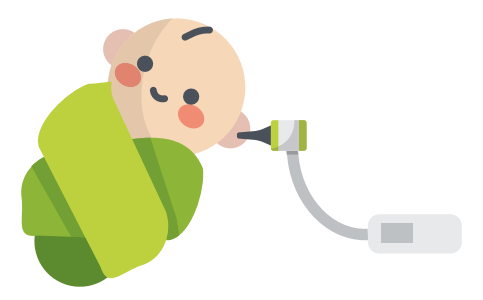

\title{
ANÁLISE DA IMPLEMENTAÇÃO DE INOVAÇÃO NO FLUXO DA TRIAGEM NEONATAL FONOAUDIOLÓGICA
}

\author{
ANALYSIS OF AN INNOVATION IN THE WORKFLOW OF THE HEARING AND
}

TONGUE NEONATAL SCREENINGS

\begin{abstract}
Luiza Aline Costa Monteiro
Fonoaudióloga. Setor de Triagem Neonatal da Maternidade Escola Januário Cicco, Universidade Federal do Rio Grande do Norte/Rede Ebserh. Discente do Mestrado Profissional em Gestão e Inovação em Saúde MPGIS/UFRN. Pesquisadora do Laboratório de Inovação Tecnológica em Saúde (LAIS) da Universidade Federal do Rio Grande do Norte. E-mail: monteirolac@gmail.com
\end{abstract}

Joseli Soares Brazorotto

Fonoaudióloga. Docente do Departamento de Fonoaudiologia da Universidade Federal do Rio Grande do Norte e do Mestrado Profissional em Gestão e Inovação em Saúde MPGIS/UFRN. Doutora em Educação Especial pela Universidade Federal de São Carlos. Pesquisadora do Laboratório de Inovação Tecnológica em Saúde (LAIS) da Universidade Federal do Rio Grande do Norte. E-mail: brazorotto@yahoo.com

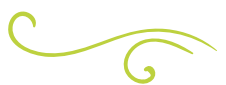

\section{RESUMO}

A triagem neonatal é realizada para a prevenção de possíveis agravos à saúde do bebê, favorecendo tratamentos precoces que diminuirão ou eliminarão fatores que possam prejudicar o desenvolvimento típico da criança. Entre os exames realizados pelo fonoaudiólogo estão os testes da orelhinha e linguinha, em que são avaliadas as condições auditiva e de movimentação da língua, respectivamente. Apesar de assegurados por lei, ambos nem sempre são realizados e, quando o são, nem sempre obedecem a um fluxo que otimize os recursos e monitore os achados, com vistas ao acompanhamento das condições de saúde do bebê. No âmbito da saúde pública, as falhas de cobertura e de gestão da informação das triagens neonatais realizadas geram impactos negativos permanentes para toda a população, com consequências nas esferas de economia e de desenvolvimento do país.
Considerou-se, pois, a importância de descrever a implementação das inovações no processo de trabalho fonoaudiológico no setor de Triagem Neonatal Fonoaudiológica de uma maternidade da Rede Ebserh, objetivo desse trabalho. Por meio de reorganização do agendamento para as triagens, mudança no local de realização dos exames e da organização de um banco de dados, foi possível obter benefícios na cobertura e no retorno financeiro para a instituição, além da melhor gestão das informações dos bebês triados. Constatou-se, pois, que a implementação da inovação no processo de trabalho fonoaudiológico trouxe melhorias efetivas ao serviço ofertado para essa população. A principal limitação do estudo referiu-se à sua característica local. Recomendam-se, pois, estudos em maternidades de outras regiões do país, considerando suas dimensões e características diversas. Futuros aprimoramentos, como a digitalização do sistema de 
agendamento e de gestão das informações, estão em estudo.

PALAVRAS-CHAVE: Triagem Neonatal, Fonoaudiologia, Inovação, Avaliação de Processos, Fluxo de Trabalho.

\section{ABSTRACT}

Neonatal screening is performed to prevent possible health problems in the baby, favoring early treatments that will reduce or eliminate factors that may impair the child's typical development. Among the tests performed by the audiologist and the speech therapist are the hearing and the tongue screenings, in which are evaluated the auditory and tongue movement conditions, respectively. Of these five screenings, the ear and tongue tests are performed by the audiologist and the speech therapist. Despite being assured by law, both are not always performed and, when they are, they do not always obey a flow that optimizes the resources and monitors the findings, in order to follow the health conditions of the baby. In terms of public health, coverage failures and information management of the neonatal screening carried out have permanent negative impacts for the entire population, with consequences in the economic and development spheres of the country. So, the aims of this study were implementing and analyzing the adoption of changes in the work process related to the flow of auditory and tongue screenings performed at a maternity hospital of the Ebserh. By reorganizing the scheduling for screening, changing the location of the examinations and organizing a database, it was possible to obtain benefits in terms of coverage and financial return for the institution, as well as better information management of the triaged babies. It was verified, therefore, that the implementation of the innovation in the process of neonatal hearing and tongue screenings brought effective improvements to the service offered to this population. The main limitation of the study was its local character. Therefore, studies are recommended in maternity hospitals in other regions of the country, considering their dimensions and different characteristics. Future enhancements, such as a scheduling and management information system are under study.

KEYWORDS: Neonatal Screening, Language and Hearing Sciences, Innovation, Process Assessment (Health Care), Workflow.

\section{INTRODUÇÃO}

O termo "triagem" significa seleção, através de testes aplicados numa população, de um grupo de indivíduos com probabilidade elevada de apresentarem determinadas patologias. A triagem neonatal é a aplicação desta metodologia de rastreamento na população entre 0 e 30 dias de vida (BRASIL, 2002).

Assim, exames neonatais são realizados no recém-nascido como forma de avaliação geral de saúde e para o diagnóstico precoce de doenças, permitindo o diagnóstico e o tratamento em tempo para diminuir ou eliminar os possíveis fatores que possam prejudicar o desenvolvimento típico da criança (MEDEIROS et al., 2016).

Entre os exames mais comuns estão os seguintes testes: orelhinha, olhinho, pezinho, coraçãozinho e linguinha, em que são avaliadas as condições auditiva, visual, sanguínea, de saturação de oxigênio e de movimentação da língua, respectivamente. Destes cinco, os testes da orelhinha e da linguinha são realizados pelo fonoaudiólogo.

Algumas considerações sobre esses testes e as condições por eles avaliadas serão abordadas no intuito de guiar a melhor compreensão do tema de estudo.

\section{Teste da Orelhinha}

A deficiência auditiva é considerada um problema de saúde pública não apenas em virtude de sua significativa prevalência, mas, 
sobretudo, pelas múltiplas consequências que pode acarretar ao desenvolvimento humano nos aspectos linguísticos, educacionais, emocionais, culturais e sociais (LIMA et al., 2015).

A incapacidade de detectar e perceber os sons, em qualquer grau, prejudica o processo de aquisição da fala e o desenvolvimento da linguagem, tão necessários à integração dos indivíduos numa sociedade cuja forma de comunicação é predominantemente oral. No mundo, cerca de 360 milhões de pessoas (aproximadamente 5\% da população mundial) vivem com deficiência auditiva incapacitante e quase 32 milhões delas são crianças (WHO, 2015). Estima-se que mais de $60 \%$ destas perdas auditivas possam ser evitadas através de medidas preventivas (WHO, 2016).

Cabe observar que a prevalência da deficiência auditiva varia de um a seis neonatos para cada mil nascidos vivos no Brasil, e de um a quatro para cada cem recém-nascidos provenientes de Unidade de Terapia Intensiva Neonatal (BRASIL, 2012). Desse modo, a Triagem Auditiva Neonatal (TAN) constitui-se como um importante processo inicial de avaliação da audição infantil que permite a detecção precoce de possíveis alterações auditivas, abrangendo todos os neonatos, inclusive aqueles que não possuem indicadores de risco para a deficiência auditiva (AMERICAN..., 2007).

Já é consenso mundial que a TAN deva ser realizada de forma universal, ou seja, que deva atingir todos os nascidos vivos (AMERICAN..., 2013). Em nosso país, desde o ano de 2010, o teste da Orelhinha é assegurado pela Lei Federal 12.303/2010 (BRASIL, 2010), por ser estratégia fundamental no planejamento das condutas clínicas e intervenções mais eficazes. Além disso, a revisão do marco normativo da Política Nacional de Triagem Neonatal (PNTN), integrando a Triagem Auditiva Neonatal (Teste da Orelhinha) a esta política, é prevista pelo Ministério dos Direitos Humanos, através do Observatório do Viver Sem Limites. Nesse sentido, estão sendo equipados hospitais e maternidades, além de terem sido elaboradas e instituídas, na Rede de Cuidados à Pessoa com Deficiência, as diretrizes de atenção para a Triagem Auditiva Neonatal (BRASIL, 2012; DEFICIÊNCIA..., 2014).

Outrossim, está prevista a reestruturação do atual sistema de busca e acompanhamento das crianças diagnosticadas com deficiência, bem como seu encaminhamento aos serviços que possam promover o tratamento necessário em cada caso.

De fato, o cuidado no acompanhamento de crianças triadas se faz primordial, pois, apesar dos avanços nos Programas de Triagem Auditiva Neonatal, reduzindo a idade média de identificação de perda auditiva, ainda há falhas nos processos de monitoramento, fazendo com que perdas auditivas tardias não sejam tratadas em tempo oportuno (NIKOLOPOULOS, 2015). Neste cenário, a busca por inovações em processos através de uma gestão eficiente, de modo a oferecer maior agilidade e maior resolubilidade, impactando na cobertura da TAN, bem como no gerenciamento dos casos identificados, faz-se importante e necessária, dado o impacto da deficiência auditiva para a sociedade.

\section{Teste da Linguinha}

O teste da linguinha foi desenvolvido no Brasil (MARTINELLI, 2012) e consiste na aplicação de um Protocolo de Avaliação do Frênulo Lingual com a finalidade de detectar a presença de alterações neste e o grau de limitação dos movimentos da língua. Essa triagem, cuja validade foi determinada em estudo nacional, irá precisar se a criança deverá ou não ser submetida à cirurgia de frenotomia, com vistas ao não comprometimento das funções de sugar, mastigar, engolir e falar (MARTINELLI, 2016).

A anquiloglossia, conhecida popularmente por "língua presa", tem uma prevalência de 1,9\% a 4,8\% (GLYNN et al., 2012) e seu impacto para a saúde infantil e, posteriormente, para a 
vida adulta, levou à obrigatoriedade da realização do teste, sendo este assegurado pela Lei no 13.002/2014 (BRASIL, 2014).

Assim como a triagem auditiva, o Teste da Linguinha tem influência na saúde e no desenvolvimento infantil, sendo outro procedimento que pode ter maior cobertura e resolubilidade com a contribuição da inovação tecnológica e de um gerenciamento eficiente.

\section{Triagem Neonatal Fonoaudiológica:}

realidade nacional e realidades locais

Apesar de assegurados por Lei, os dois testes ainda se apresentam incipientes em nosso país, com estudos referindo uma cobertura insuficiente, por exemplo, da triagem auditiva neonatal (DIAS; PASCHOAL; CAVALCANTI, 2017; PASCHOAL; CAVALCANTI; FERREIRA, 2017), com a taxa nacional de cobertura de $37,2 \%$, bastante distante da recomendação de mais de 95\% de acesso à TAN (LEWIS et al., 2010). Outrossim, ainda há a necessidade de desenvolvimento de um protocolo padrão quanto à gestão dos processos da triagem neonatal fonoaudiológica, os quais vão desde a identificação dos pacientes, passando pela realização dos exames, orientação à família, busca ativa em caso de falta e acompanhamento nos casos em que há necessidade de monitoramento (NIKOLOPOULOS, 2015; LEO et al., 2016). Para ambas as triagens há recomendações nacionais quanto ao fluxo dos procedimentos e encaminhamentos (BRASIL, 2012; BRASIL, 2016).

Entretanto, considerando a ausência de um protocolo nacional de gestão dos processos e das informações da triagem fonoaudiológica em maternidades, muitos serviços podem ter inúmeras problemáticas como: baixa cobertura, alta taxa de reteste e ausência de registro dos exames, além do possível subaproveitamento dos recursos de modo geral (tanto das finanças, quanto dos equipamentos e dos recursos humanos).

A variabilidade destes problemas, somada à heterogeneidade na condução dos Programas de Triagem Neonatal, compromete não apenas a qualidade dos referidos programas de maneira local, mas também interfere negativamente na comparação entre os programas, no planejamento de ações específicas e na melhoria contínua da qualidade dos serviços (LEO et al., 2016). No segundo semestre de 2014, esta realidade foi diagnosticada no serviço de Triagem Neonatal Fonoaudiológica da Maternidade Januário Cicco da Universidade Federal do Rio Grande do Norte, gerenciada pela Rede Ebserh.

Frente a tal diagnóstico foi organizado um fluxo no processo de triagem neonatal fonoaudiológica de modo a minimizar perdas e maximizar recursos, melhorando sua eficiência, em consonância com os princípios do Sistema Único de Saúde, o que será descrito na seção métodos.

Tais mudanças no fluxo constituíram-se como estratégias de inovação na saúde, com as características de melhorar seu desempenho, agregar novos conhecimentos, manter a sustentabilidade, medir seus indicadores e buscar suporte em tecnologias, garantindo assim um serviço com mais eficiência (NODARI et al., 2015; CANHA, 2016; RIVERA; ARTMANN, 2016).

Para Winston-Gerson e Roush (2016) um programa bem executado não se restringe à técnica do procedimento em si, mas inclui, dentre outros elementos, a aplicação de protocolos bem definidos e a manutenção de boas práticas, como o monitoramento dos indicadores, por exemplo. Nesse sentido, o presente artigo tem por objetivo descrever a implementação de inovação no processo de trabalho fonoaudiológico no setor de Triagem Neonatal Fonoaudiológica de uma maternidade da Rede Ebserh. 


\section{MATERIAIS E MÉTODOS}

Trata-se de um estudo analítico e descritivo, dividido em etapas, mencionadas a seguir:

\section{Etapa I - Análise Situacional}

Considerando a importância dos testes da orelhinha e linguinha, foram detectados, no segundo semestre de 2014, os seguintes problemas no Setor de Triagem Fonoaudiológica:

- Índice de reteste do Teste da Orelhinha superior a $20 \%$;

- Comprometimento na cobertura pela saída diária de recém-nascidos da maternidade sem que os testes da orelhinha e da linguinha fossem realizados;

- Dificuldade em monitorar as informações sobre os resultados dos exames;

- Perda de tempo em decorrência da logística necessária para realizar os procedimentos em cada enfermaria (total de 4, em prédios diferentes);

- Atendimento feito antes da alta hospitalar e, consequentemente, sem repasse financeiro para a instituição quanto à realização desses procedimentos.

\section{Etapa Il - Mudanças Propostas a partir da Pergunta Norteadora}

A partir da pergunta: "Como otimizar o serviço de triagem fonoaudiológica com os recursos de que já dispomos?" foi proposta a implementação de inovação para o fluxo do atendimento (alteração no momento e no local dos testes) e o uso de um banco de dados para o gerenciamento das triagens, mantendo os mesmos recursos (materiais e humanos). Foram propostas, pois, as seguintes mudanças:
A. Alteração no momento dos testes: ao invés de realizar os exames antes da alta hospitalar dos recém-nascidos, seriam realizados por agendamento (pós-alta, dentro do período recomendado de até 30 dias do bebê);

B. Alteração do local dos testes: ao invés de alternar locais de atendimentos entre as enfermarias, todos os bebês passariam a ser triados em sala mais adequada, exclusiva para os procedimentos de triagem fonoaudiológica;

C. Alteração na aquisição e no monitoramento de dados dos testes: organização do banco de dados para monitorar os índices de cobertura, faltas e organização dos agendamentos.

\section{Etapa III - Organização das Etapas e dos Procedimentos}

O processo de reorganização dos procedimentos e do fluxo de atendimento para os testes da orelhinha e da linguinha se deu da seguinte forma (vide Fluxograma 1):

\section{Análise do Prontuário}

Inicialmente, foi feita, em cada enfermaria, uma análise dos prontuários com o intuito de registrar na anamnese os indicadores para a perda auditiva. Este momento é fundamental, pois há informações de que as mães/responsáveis podem não lembrar ou omitir no momento do exame, e a ausência de informações pode interferir na conduta pós-teste. Por exemplo, se houver um único indicador de risco (hiperbilirrubinemia) e este não for citado, a conduta adotada será "alta", quando deveria ser "monitoramento auditivo".

Através do prontuário também foram colhidas informações da genitora relacionadas à gestação e à sua identificação (como nome completo, número do cartão SUS, cidade origem, telefone, idade, número de filhos, profissão, escolaridade e estado civil). 


\section{Agendamento}

O agendamento foi feito por uma ficha de papel, entregue à mãe ou ao responsável, com a data e horário do exame de triagem, e o agendamento foi anotado em planilha Excel®. O agendamento ocorre da seguinte forma: através de uma agenda no computador, a data para realização dos exames foi selecionada. Após isto, a genitora recebe em leito um "comprovante de agendamento". Na ocasião, a mãe do recém-nascido foi devidamente orientada sobre a importância dos testes para o seu filho.

\section{Realização dos Exames}

$\mathrm{Na}$ data agendada, a mãe comparece com o(a) filho(a) para realizar os dois testes. O fonoaudiólogo deve, então, confirmar os dados, realizar outras perguntas que julgar pertinentes e iniciar a execução dos procedimentos. Após isto, deve anotar os dados na caderneta de saúde da criança e no prontuário que fica sob a guarda do serviço, além de prestar as devidas informações para a mãe e/ou os responsáveis. Em quaisquer dos testes, se houver necessidade de reteste, os registros também serão feitos (na caderneta e no prontuário) com as devidas informações. Ao final dos exames, pais/responsáveis devem receber o resultado dos testes e todas as orientações relacionadas a eles, ainda que as respostas sejam favoráveis em ambos os testes. Sugere-se, neste momento, prestar ainda orientações sobre o desenvolvimento da audição e da linguagem infantil, como importante estratégia para o contínuo monitoramento, pela família, do desenvolvimento da criança.

\section{Banco de Dados}

Todos os achados, após o preenchimento do prontuário do serviço (anamnese fonoaudiológica), alimentam um banco de dados em planilha do Excel $\circledast$, o qual fornece dados relevantes sobre a quantidade de bebês com alteração nos testes da oreIhinha/linguinha (como cidade de origem, entre outros) bem como quanto aos principais indicadores de risco para a perda auditiva.

\section{Análise dos achados}

No momento, os achados dos testes realizados na maternidade estudada encontram-se cadastrados em banco de dados Excel®. A partir deles, é possível avaliar os indicadores propostos pelo serviço, permitindo condutas mais acertadas, além da realização de pesquisas na área, na constante busca pela melhoria da qualidade do serviço. Ressalta-se, no entanto, que a carga-horária para realizar tais análises é contabilizada no horário de atendimento do fonoaudiólogo.

\section{Faturamento dos exames}

A partir da planilha utilizada para registro dos exames, são contabilizados a quantidade de exames feitos e encaminhados para o setor responsável (Faturamento/ Financeiro) e o quantitativo de cada exame com as informações que o próprio setor solicita (nome genitora/cartão SUS) a fim de que a Instituição receba os recursos financeiros pela realização dos procedimentos.

O pagamento é feito através de recursos advindos do FAEC - Fundo de Ações Estratégicas e Compensação. Os procedimentos têm seus códigos cadastrados no SIGTAP - Sistema de gerenciamento da tabela de procedimentos, medicamentos e OPM do SUS -, sendo utilizados pelo setor financeiro para que o pagamento seja feito. 


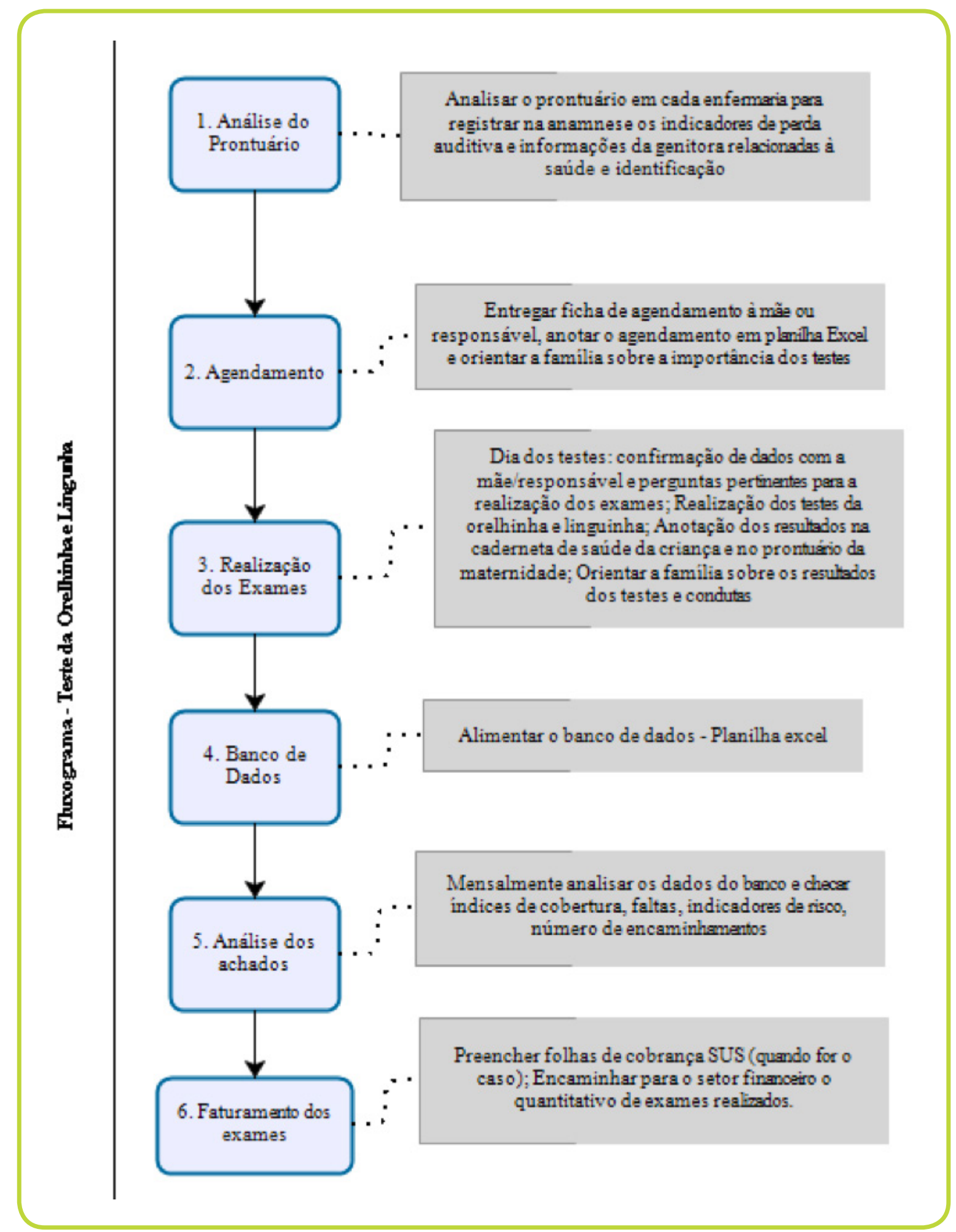

Fluxograma 1 - Descrição das etapas do processo de trabalho após a implementação da inovação.

Fonte: Autoria própria (2018).

Após 3 meses da implementação da inovação no fluxo de trabalho (agendamento, local de realização dos exames e banco de dados), foi realizada nova análise, a qual será apresentada na seção Resultados. 


\section{RESULTADOS}

Após três meses de teste com a implementação da inovação no processo de trabalho do fonoaudiólogo na Triagem Neonatal Fonoaudiológica (com realização dos exames por agendamento e organização do banco de dados), foi possível observar:

- Índice de reteste do Teste da Orelhinha inferior a 3\%;

- Atendimentos realizados em uma única sala, com a otimização de tempo, dobrando a capacidade de atendimentos/turno, além da melhoria na condição da TAN e de espaço mais adequado para a orientação às famílias;

- Maior controle dos bebês para realização dos testes da orelhinha e da linguinha, visto que saíam com agendamento marcado, ampliando assim a cobertura;

- Tempo suficiente para a busca ativa em caso de falta (cooperando no controle da cobertura);

- Monitoramento do absenteísmo e dos casos atendidos, a partir de um banco de dados em Excel $\circledast$ alimentado diariamente;

- Mudança nos atendimentos, que passaram a ser feitos de forma "ambulatorial", podendo, então, ser faturados para a instituição com recursos advindos do FAEC.

- Sobre estas observações, vale ressaltar:

\section{Índice de Reteste - Teste da Orelhinha}

As alterações do momento e do local da realização deste teste modificaram os índices de cobertura e reteste, importantes para o bom funcionamento de um programa de triagem auditiva neonatal.
O teste da Orelhinha é realizado por meio de equipamentos eletrônicos para a captação de respostas do sistema auditivo do bebê e requer algumas condições mínimas para a qualidade do resultado da triagem. Antes da proposta de inovação (envolvendo mudança no processo de trabalho), fatores como a presença de secreções no conduto auditivo do neonato e o ruído externo interferiam na execução e no resultado desse teste.

Efetivamente, a quantidade de reteste nos exames do Teste da orelhinha, que chegou a 24\%, caiu para índices inferiores a $3 \%$, o qual se mantém até a presente data. Acredita-se que essa importante redução ocorreu em virtude de fatores como:

- A redução da secreção no conduto auditivo proveniente do parto (é comum que ela ainda esteja no conduto auditivo em menos de 48hs), pois o agendamento se dá de 10 a 15 dias após o nascimento, dentro do prazo recomendado para a TAN (primeiro mês de vida nos neonatos ou até o terceiro mês de idade corrigida, considerando prematuros e aqueles com longos períodos de internação);

- A diminuição do ruído no momento do teste pela utilização de local único e mais adequado (sala de triagem fonoaudiológica), gerando menos resultados falsos-positivos, e, portanto, menor índice de reteste.

\section{Controle da Cobertura e Busca Ativa}

Anteriormente, os exames começavam a ser feitos em uma enfermaria, sendo comum que, concomitantemente, estivesse havendo alta hospitalar em uma outra enfermaria, ocasionando a saída de bebês não triados da maternidade, sem controle da quantidade de neonatos ainda não triados. A partir do processo por agendamento, todos os neonatos são agendados e, portanto, há maior controle sobre a taxa de cobertura.

Além disso, com o agendamento dos exames, foi possível controlar os atendimentos 
diários e verificar o quantitativo de presença e falta. A cobertura em média é de $90 \%$, tendo já chegado a $97 \%$ de cobertura, índice dentro do ideal recomendado (acima de $95 \%)$.

O absenteísmo é controlado através da busca ativa. Em caso de falta, como há previamente o contato registrado da mãe (pela análise do prontuário no período de internação na maternidade), os fonoaudiólogos ligam para a genitora e marcam uma nova data para que os testes sejam feitos. Isso é repetido para até três faltas.

\section{Banco de Dados}

Por conta do banco de dados que é alimentado após cada exame feito, é possívelmonitorar todos os achados, cooperando para a produção científica e a formação de alunos residentes. Antes, isso não era possível.

No Quadro 1, a seguir, foram organizados os resultados da implementação da inovação no processo de trabalho do fonoaudiólogo, comparando antes e após 3 meses da modificação descrita.

\begin{tabular}{|c|c|c|}
\hline & INOVAÇÃO & DEPOIS \\
\hline CRITÉRIO ANALISADO & ANTES & Menor que 3\% \\
\hline Reteste - Teste da Orelhinha & Maior que 20\% & Total \\
\hline Controle dos bebês nas enfermarias & Inexistente & 90\% \\
\hline Cobertura dos Testes & Sem estatística & Total \\
\hline Monitoramento dos Achados & Inexistente & 20 \\
\hline Quantidade de atendimentos/turno & 10 & Diária \\
\hline Busca Ativa & Inexistente & Faturamento de todos os \\
\hline Retorno Financeiro & Sem faturamento & atendimentos \\
\hline
\end{tabular}

Quadro 1 - Resultados da implementação da inovação no processo de trabalho do fonoaudiólogo na Triagem Neonatal Fonoaudiológica.

Fonte: Autoria própria (2018).

\section{DISCUSSÃO}

A inovação, tanto tecnológica quanto organizacional, tem trazido incontáveis benefícios para o Sistema de Saúde, uma vez que tem sido vista como a solução para que ele se torne capaz de responder aos novos desafios que enfrenta. As mudanças implementadas no fluxo de trabalho fonoaudiológico na maternidade estudada nesta pesquisa melhoraram, pois, a qualidade do processo produtivo, tornando o serviço de triagem fonoaudiológica neonatal mais eficiente (NODARI et al., 2015; RIVERA; ARTMANN, 2016; CANHA, 2016).

Destaca-se que a triagem, seguida do diagnóstico e da intervenção precoces referentes à deficiência auditiva (LIMA etal., 2015, WHO, 2015; WHO, 2016) e à anquiloglossia (GLYNN et al., 2012; MARTINELLI, 2012; MARTINELLI, 2016), são ações fundamentais para a garantia da saúde de bebês e de suas famílias. A implementação da inovação no processo de triagem dessas condições poderá, pois, minimizar ou mesmo eliminar as consequências de tais problemas. Nesse sentido, deve-se estimular a realização de 
pesquisa em serviços de triagem neonatal, característica essencial deste estudo.

A partir da análise situacional da triagem fonoaudiológica neonatal na maternidade estudada e da pergunta norteadora, foi possível identificar e adotar a possibilidade do agendamento ambulatorial dos testes como uma inovação no fluxo de trabalho do fonoaudiólogo, sem a necessidade de investimentos extras em recursos humanos ou financeiros (RIVERA; ARTMANN, 2016).

A partir do atendimento por agendamento foi possível, então, aumentar a quantidade de atendimentos, repercutindo na melhora da cobertura, a qual não possuía controle, pois enquanto alguns neonatos eram atendidos numa enfermaria, simultaneamente a alta hospitalar ocorria em outra enfermaria (prédio anexo), de modo dificultar o controle de cobertura. Com a mudança, em alguns meses, foi possível praticamente alcançar o que preconizam as Diretrizes de Atenção à TAN, bem como, na maioria dos meses, ter uma média de $90 \%$ de cobertura, muito próxima às recomendações e acima das estatísticas da região nordeste e mesmo da cobertura nacional para o teste da orelhinha. Destaca-se que, como o teste da linguinha é mais recente, não há estudos que façam referência ao seu índice de cobertura nacional e/ou por regiões no Brasil (BRASIL, 2010; LEWIS et al., 2010; BRASIL, 2012; BRASIL, 2014; DEFICIÊNCIA..., 2014; BRASIL, 2016; NIKOLOPOULOS, 2015; DIAS; PASCHOAL; CAVALCANTI, 2017; PASCHOAL; CAVALCANTI; FERREIRA, 2017).

Outra mudança advinda do agendamento foi a alteração do local em que eram realizados os testes. Aparentemente, esse é um ganho secundário, contudo, denotou-se melhora na qualidade do ambiente de teste para a triagem auditiva (é necessário um ambiente silencioso para a execução do mesmo, conforme LEWIS et al., 2010, BRASIL, 2012), com um espaço mais acoIhedor, o qual propiciou maior atenção das famílias para as orientações fonoaudiológicas e maior tranquilidade e privacidade nos atendimentos. Como destacado pelo estudo de Nikolopoulos (2015), é essencial que as famílias possam confiar nas orientações para prosseguirem com o monitoramento auditivo de seus filhos (em casos em que há indicador de risco para a perda auditiva). Para minimizar essa possibilidade de não seguimento, os pais cujos neonatos precisam de acompanhamento são devidamente orientados, ainda em sala, após exames, sobre a necessidade e importância do monitoramento auditivo.

Ressalta-se que a possibilidade de realizar o teste da orelhinha por meio de agendamento e em ambiente silencioso foi determinante para a diminuição do alto índice de reteste (20\%) para aproximadamente 3\%, conforme o preconizado para a TAN (LEWIS et al., 2010; BRASIL, 2012), sendo este um ganho específico para a triagem auditiva neonatal, importante para a melhoria da qualidade do serviço.

Além das diretrizes (BRASIL, 2012), a literatura enfatiza a importância de que os Serviços de Triagem Auditiva Neonatal utilizem um banco de dados que permita, além da atenção quanto à cobertura, o controle do índice de reteste, de encaminhamentos e de falsos-positivos. Tal controle visa fornecer índices de qualidade do serviço (LEO et al., 2016) e possibilita futuros estudos de prevalência das condições avaliadas, importantes para a gestão da saúde neonatal e infantil em nosso país (BRASIL, 2002; MEDEIROS et al., 2016).

Assim, os resultados obtidos com a implementação do novo processo adotado no Serviço de Triagem Neonatal Fonoaudiológica da Maternidade Escola Januário Cicco ratificam o valor da inovação para o sistema de saúde e corroboram com a importância de garantir programas eficazes e eficientes (WINSTON-GERSON; ROUSH, 2016). Além disso, cooperam para o emprego das recomendações oficiais na organização dos serviços de triagem fonoaudiológica no Brasil (BRASIL, 2012; BRASIL, 2016), bem como daquelas destacadas pela literatura e comitês científicos (AMERICAN..., 2007, 
AMERICAN..., 2013) para a melhoria contínua da qualidade (NIKOLOPOULOS, 2015; LEO et al., 2016).

\section{CONSIDERAÇÕES FINAIS}

A inovação implementada no fluxo da triagem neonatal fonoaudiológica referente ao agendamento de atendimentos, ao local de realização dos testes e à aquisição e ao monitoramento dos dados da triagem se refletiu em índices positivos, culminando na melhoria da qualidade do serviço na maternidade estudada. Em virtude de todos os benefícios encontrados, considerou-se importante mapear o processo, a fim de que o mesmo possa posteriormente ser apresentado para outras unidades hospitalares.

A principal limitação do estudo referiu-se à sua característica local, muito embora sejam fundamentais as pesquisas em serviço, particularidade do referido trabalho. Nesse sentido, a motivação para a análise e a implementação da inovação no serviço trouxeram melhorias em pouco tempo de experienciação com o novo modelo de fluxo de trabalho, o que também retroalimentou a pesquisa, com possibilidade da aquisição de dados.

Outra limitação referiu-se ao caráter descritivo do estudo, que carrega em si possibilidades de outras análises e desenhos metodológicos, desejáveis para futuras pesquisas conduzidas na área. Pesquisas multicêntricas, por exemplo, empregando uma ou outra inovação já proposta, a combinação destas, ou ainda novos arranjos ou inovações tecnológicas, com delineamento do tipo experimental e análise estatística inferencial, podem trazer soluções a serem empregadas nacionalmente, com chances de sucesso.

Destaca-se que esta pesquisa se encontra em andamento no intuito de trazer constantes melhorias ao serviço em questão e que já foram desenvolvidas outras duas etapas, a saber: inicialmente, a análise situacional da triagem neonatal fonoaudiológica na Rede
Ebserh e, posteriormente, a elaboração e o lançamento de um sistema digital de agendamento e gerenciamento dos dados da triagem fonoaudiológica neonatal, a ser implementado na sequência, para estudos posteriores.

Assim, recomenda-se a continuidade das pesquisas na área da triagem neonatal, neste caso, em especial, Testes da Orelhinha e Linguinha. Em seus vários aspectos, a realização destes testes conta com possibilidades de aprimoramento, desde o planejamento da triagem, das condições em que são realizados os exames, até a melhoria do custo-efetividade dos procedimentos de triagem, envolvendo a orientação fonoaudiológica às famílias dos bebês triados e o monitoramento desses bebês, tendo em vista que a triagem é apenas a entrada para o neonato no sistema de saúde.

As perspectivas futuras quanto ao emprego da inovação, seja na tecnologia ou no gerenciamento dos serviços, são, portanto, promissoras para a Triagem Neonatal Fonoaudiológica no Brasil, cooperando com a prestação de um serviço de excelência para o usuário do Sistema Único de Saúde. 


\section{REFERÊNCIAS BIBLIOGRÁFICAS}

AMERICAN ACADEMY OF PEDIATRICS. Joint Committee on Infant Hearing. Year 2007 position statement: principles and guidelines for early hearing detection and intervention programs. Pediatrics, v. 120, n. 4, p. 898-921, out. 2007.

AMERICAN ACADEMY OF PEDIATRICS. Joint Committee on Infant Hearing. Supplement to the JCIH 2007 Position Statement: Principles and Guidelines for Early Intervention After Confirmation That a Child Is Deaf or Hard of Hearing. Pediatrics, v. 131, n. 4, p. 1324-1349, abr. 2013.

BRASIL. Ministério da Saúde. Secretaria de Assistência à Saúde. Coordenação-Geral de Atenção Especializada. Manual de Normas Técnicas e Rotinas Operacionais do Programa Nacional de Triagem Neonatal. Brasília: Ministério da Saúde, 2002.

BRASIL. Ministério da Saúde. Secretaria de Assistência à Saúde. Departamento de Ações Programáticas Estratégicas. Diretrizes de Atenção da Triagem Auditiva Neonatal. Brasília: Ministério da Saúde, 2012.

BRASIL. Lei n 12.303, de 02 de agosto de 2010. Obriga a realização do exame denominado Emissões Otoacústicas Evocadas. Disponível em: <http://www.planalto.gov.br/ccivil_03/_Ato2007-2010/2010/Lei/ L12303.htm>. Acesso em: 14 ago. 2018.

BRASIL. Lei n 13.002, de 20 de junho de 2014. Obriga a realização do protocolo de avaliação do Frênulo da língua em bebês.

Disponível em: <http://www2.camara.leg.br/legin/fed/lei/2014/lei13002-20-junho-2014-778947-publicacaooriginal-144433-pl.html>. Acesso em: 14 ago. 2018.

BRASIL. Ministério da Saúde. Secretaria de Atenção à Saúde. Departamento de Ações Programáticas Estratégicas. Coordenação Geral de Saúde da Criança e Aleitamento Materno. Nota Técnica no 09/2016. Disponível em: <http://www.redeblh.fiocruz.br/media/ notatecn9_16.pdf>. Acesso em: 14 ago. 2018.

CANHA, J. O Valor da Inovação: Criar o Futuro do Sistema de Saúde. Cad. Saúde Pública, Rio de Janeiro, v. 32, n. 2, 2016.

DEFICIÊNCIA Viver sem Limite - Plano Nacional dos Direitos da Pessoa com / Secretaria de Direitos Humanos da Presidência da República (SDH/PR). VIVER SEM LIMITE - Plano Nacional dos Direitos da Pessoa com Deficiência: SDH-PR/ SNPD, 2014. 180 p. 
DIAS, W. C. F. G. S.; PASCHOAL, M. R.; CAVALCANTI, H. G. Analysis of the coverage of neonatal hearing screening in Northeast Brazil. Audiol Commun Res. v. 22, e1858, p. 1-7, 2017.

GLYNN, R. W. et al. Division of tongue tie: Review of pratice trought a tertiary paediatric otorhinolaryngology service. International Journal of Pediatric Otorhinolaryngology. 2012.

LEWIS, D. R. et al. Comitê multiprofissional em saúde auditiva COMUSA. Braz J Otorhinolaryngol, v. 76, n. 1, p. 121-128, 2010.

LEO, C. G. et al. A conceptual framework for rationalized and standardized Universal Newborn Hearing Screening (UNHS) programs. Italian Journal of Pediatrics, v. 42, n. 15, 2016.

LIMA, P. T. et al. A triagem auditiva neonatal na Rede Municipal do Rio de Janeiro, Brasil. Ciênc. Saúde Coletiva, Rio de Janeiro, v. 20, n. 1, p. 57-63, jan. 2015.

MARTINELLI, R. L. C. et al. Protocolo de avaliação do frênulo lingual para bebês: relação entre aspectos anatômicos e funcionais. Rev. CEFAC, São Paulo, v. 14, n. 1, p. 138-145, jan./fev. 2012.

MARTINELLI, R. L. C. et al. Validade e confiabilidade da triagem: "teste da linguinha". Rev. CEFAC, v. 18, n. 6, São Paulo, nov./dec. 2016.

MEDEIROS, H. E. G. B. de et al. Sistema de apoio à decisão na realização e acompanhamento do teste da linguinha. Revista Brasileira de Computação Aplicada, v. 8, n. 1, p. 104-113, abr. 2016.

NIKOLOPOULOS, T. P. What we have achieved and what needs to be improved. International Journal of Pediatric Otorhinolaryngology, v. 79, issue 5, p. 635-637, may. 2015.

NODARI, C. H. et al. Configuração das práticas de inovação na atenção primária à saúde: estudo de caso. Ciênc. Saúde Coletiva, Rio de Janeiro, v. 20, n. 10, p. 3073-3086, oct. 2015.

PASCHOAL, M. R.; CAVALCANTI, H. G.; FERREIRA, M. A. F. Spatial and temporal analysis of the coverage for neonatal hearing screening in Brazil (2008-2015). Ciência \& Saúde Coletiva, v. 22, n. 11, p. 36153624, 2017.

RIVERA, F. J. U.; ARTMANN, E. Innovation and communicative action: health management networks and technologies. Cad. Saúde Pública, Rio de Janeiro, v. 32, supl. 2, p. 1-11, 2016. 
WHO. World Health Organization. 2015. Prevention of blindness and deafness: estimates. Geneva. 2015. Disponível em: <http://www.who. int/pbd/deafness/estimates/en/>. Acesso em: 11 nov. 2017.

WHO. World Health Organization. Deafness and hearing loss factsheet. Geneva. 2016. Disponível em: <http://www.who.int/ mediacentre/factsheets/fs >. Acesso em: 11 nov. 2017.

WINSTON-GERSON, R.; ROUSH, J. Outsourcing Hospital-Based Newborn Hearing Screening: Key Questions and Considerations. The Journal of Early hearing detection and intervention. v. 1, issue 1, p. 21-25, 2016. 